\title{
9
}

Antonio López Arce*

\section{LA INVERSIÓN ESPAÑOLA EN CHINA EL CASO GESTAMP}

El sector de la automoción vive desde hace décadas un crecimiento constante en China. Así, las perspectivas para el sector de componentes en general y para Gestamp en particular pasan por seguir creciendo en este mercado, de la mano de los clientes extranjeros, pero también con los locales. En este sentido, Gestamp acompañará el crecimiento de sus clientes con inversiones en el país encaminadas a cubrir la demanda del mercado. Todo con un objetivo: realizar carrocerías, chasis y mecanismos cada vez más ligeros y seguros que reduzcan el impacto ambiental de los vehículos al aligerar su peso y los hagan más seguros. China es, para Gestamp, una zona prioritaria dentro de su plan estratégico al ser uno de los mercados en los que se concentra el crecimiento de la fabricación de vehículos.

Palabras clave: fabricación de automóviles, inversión extranjera.

Clasificación JEL: F21, L62.

\section{Introducción}

Es importante destacar los significativos esfuerzos que ha estado realizando China durante los últimos años en cuanto a la apertura al exterior, fundamentalmente a nivel comercial. EI XIII Plan Quinquenal 2016-2020, aprobado en marzo de 2016 durante la XII Asamblea Popular Nacional, y por el que se guían las políticas de actuación del Gobierno, está diseñado para hacer frente a los serios problemas que presenta la economía china de un crecimiento desequilibrado, descoordinado e insostenible, y propone un modelo de desarrollo basado en la innovación,

*Chief Financial Officer de Gestamp en Asia.

Versión de diciembre de 2017 respetuoso con el medio ambiente y más abierto al exterior.

Gracias a las políticas del Gobierno y a la apertura del país, China está experimentando un continuo crecimiento de su economía. No obstante, es conveniente destacar la ralentización en la tasa de crecimiento, consecuencia de las reformas estructurales que se vienen aplicando en el país desde 2013 para transformar el patrón de crecimiento hacia un modelo más sostenible.

Debido a esta corriente aperturista, el régimen de inversiones extranjeras que regula el mercado chino está actualmente en revisión. En líneas generales, se favorecen los sectores de alta tecnología, nuevas energías e I+D; se incentivan los sectores que incorporen ahorro energético y protección medioambiental; y $\triangleright$ 
se produce una cierta apertura en algunos sectores de servicios, entre otros aspectos. Esto favorece enormemente a la industria de la automoción, considerada por el Gobierno chino como una de las más importantes del país.

El marco global de las inversiones extranjeras en China se basa principalmente en una ley general en proceso de revisión y que sustituye a las tres actuales vigentes, un nuevo sistema de aprobación mediante registro electrónico y el Catálogo de Industrias de Guía de Inversión Extranjera, que fue publicado por primera vez en 1995 y que contiene tres categorías de sectores objeto de inversión extranjera (favorecidos, restringidos y prohibidos).

\section{El sector del automóvil en China}

\subsection{Un mercado en crecimiento}

La industria de la automoción en China ha experimentado una gran expansión en los últimos años. Si bien el desarrollo de esta industria en el país se inició a principios de los años noventa, es a partir de 2001, año en el que China accede a la Organización Mundial del Comercio, cuando realmente se produce un progreso acelerado. Entre 2002 y 2007 , el mercado del automóvil en China creció a un promedio del 21 por 100 anual, equivalente a un millón de vehículos nuevos cada año. En 2007, produjo más de ocho millones de automóviles. En 2009, la producción alcanzó los 13,7 millones de automóviles, de los cuales 10,3 millones eran turismos y 3,4 millones, vehículos comerciales, superando a Estados Unidos como el mayor productor de automóviles del mundo por volumen, posición que no ha abandonado desde entonces y que está sostenida en mayor parte por la sólida demanda de marcas extranjeras. Ya en 2015, la producción anual de vehículos de motor alcanzó los 24,5 millones de unidades.

Detrás del espectacular desarrollo de la industria del automóvil en China se encuentran diferentes factores, entre los que cabe citar el fuerte y sostenido crecimiento económico del país, el creciente poder adquisitivo de las familias y el desarrollo de las clases medias, el importante desarrollo de la red de carreteras y autopistas $^{1}$, así como el creciente número de fabricantes locales y multinacionales del automóvil (europeos, norteamericanos, japoneses y coreanos) dispuestos a invertir en China.

De acuerdo con las previsiones de los expertos, se espera que estos factores sigan impulsando la demanda en los próximos años. Además, cabe destacar que la industria de la automoción ha sido históricamente un sector clave para el Gobierno chino. En la actualidad, concretamente, es el más importante del país y continuará creciendo durante los próximos años gracias, entre otros factores, a los programas de estímulo del Gobierno chino, como el XIII Plan Quinquenal 2016-2020.

\subsection{Composición actual}

En los últimos años, las marcas chinas de vehículos de pasajeros han ido perdiendo cuota de mercado en China frente a los competidores extranjeros (ha caído desde el 60 por 100 en 2010 hasta el 49 por 100 de 2015). Todo ello aun cuando estos últimos están obligados por la legislación china a formar joint-ventures con empresas locales y a limitar su participación $\triangleright$

\footnotetext{
1 La red de autopistas, que ya es la segunda del mundo, ha ido creciendo desde el año 2000 a una media de un 20 por 100 anual y se estima que se multiplicará por 1,5 en la próxima década. Aun así, la red de carreteras es todavía insuficiente para la cantidad de coches que los ciudadanos chinos están comprando (Oficina Económica y Comercial de España en Pekín: Informe económico y commercial, febrero de 2017).
} 
al 50 por 100 del capital. El principal objetivo que persigue esta medida es que las firmas extranjeras compartan parte de su conocimiento técnico con sus socios, pero la realidad es que la transferencia de conocimiento no está consiguiendo los resultados que el Gobierno chino esperaba. Una de las posibles explicaciones que barajan los expertos es que las empresas chinas aún son tecnológicamente débiles y poseen menor reputación de marca que los fabricantes extranjeros. Cabe destacar que se ha producido un cambio en los objetivos de posicionamiento de los productores locales, que quieren situarse como oferentes de calidad y valor añadido frente a solo precios baratos. En este sentido, han hecho esfuerzos para mejorar sus productos. Sin embargo, sus competidores internacionales también han continuado avanzando, cargando sus modelos con tecnologías de navegación innovadoras, equipamiento de entretenimiento y seguridad más avanzada, lo que les permite seguir manteniendo su posición de liderazgo frente a los locales.

Ante esta situación, algunas compañías chinas están optando por adquirir participaciones en firmas extranjeras (MG Rover, PSA, Fisker, Volvo o Saab, por ejemplo) que les aporten la tecnología, el conocimiento y la visibilidad en el mercado que no han conseguido hasta ahora.

Actualmente, el mercado de automóviles chino está dominado por empresas mixtas que producen modelos de Volkswagen, Citroën, Chrysler, Daihatsu, Honda, Nissan, Ford, Mazda, Toyota, GM, Fiat, Hyundai, BMW y Renault. Sin embargo, en los últimos años, grupos como SAIC, FAW, Beijing Automotive Industry, Donfeng Motor Group y Guangzhou Automotive han creado marcas domésticas propias en línea con la política del Gobierno de promover este tipo de iniciativas, y siguiendo el ejemplo de los dos principales fabricantes independientes chinos, Chery y Geely. Esta última adquirió en 2010 la americana Volvo por 1.800 millones de dólares. A finales de 2007 , el mayor fabricante de automóviles chino, Shanghai Automotive Industry Corporation (SAIC), firmó un acuerdo para comprar el negocio de ensamblaje y componentes de uno de sus principales rivales, Nanjing Auto Corporation (NAC). En esta línea, han continuado produciéndose más movimientos de absorción o fusión en la industria.

Con ello, los compradores chinos pueden elegir entre una oferta de marcas y automóviles más amplia que en cualquier otro país. En mayo de 2016, había 130 marcas de automóviles disponibles en China, divididas en tres tipos: las marcas extranjeras, las marcas chinas locales y las submarcas provenientes de las empresas conjuntas entre firmas extranjeras y chinas. No obstante, la industria automovilística en China se mantiene muy concentrada. Según los últimos datos disponibles, el top 10 de los 70 fabricantes existentes representaba en torno al 89 por 100 de las ventas totales en 2013, subiendo desde el 87 por 100 del año anterior. Ante esta realidad, el Gobierno chino se ha posicionado repetidas veces a favor de una consolidación de la industria, pero tanto gobernadores locales como nacionales temen que la pérdida de empleos resultante pudiera generar inestabilidad. Por ello, el progreso en este ámbito ha sido lento hasta ahora.

\subsection{Retos del mercado automovilístico chino}

Teniendo en cuenta la posición consolidada del vehículo convencional en China, a continuación se exponen los principales retos que deberá afrontar el sector automovilístico en los próximos años. 
1. Necesidad de cumplir con los estándares internacionales de calidad y seguridad para tener una mayor presencia en países desarrollados.

2. La gama alta. El sector de los vehículos de lujo está en auge en China debido al aumento del poder adquisitivo de los consumidores y al cambio en sus preferencias; apreciando cada vez más la calidad, el diseño y las prestaciones de los vehículos de este tipo. De este modo, se espera que las ventas en este segmento crezcan en los próximos años con tasas mucho mayores a las del mercado automotriz general en el país. Por otro lado, la demanda de coches de lujo está siendo impulsada por la creciente población joven y rica de la nación; en particular, varones de entre 25 y 35 años, que buscan afianzar su estatus de cara a la sociedad. Para 2020 habrá 300 ciudades en China que tendrán consumidores con suficientes ingresos para comprar automóviles premium, según un informe publicado por la consultora McKinsey².

3. Vehículos impulsados por energías alternativas. El crecimiento económico amigable con el planeta, es decir, atendiendo al cuidado del medio ambiente, es uno de los objetivos destacados en el XIX Congreso del Partido Comunista Chino celebrado en octubre de 2017. No es de extrañar teniendo en cuenta que según el informe State of Global Air $2017^{3}$, en India y en China se produjeron

\footnotetext{
2 McKinsey \& Company (2013, marzo). Upward mobility: The future of China's premium car market. Disponible en: https://www. mckinsey.com/industries/automotive-and-assembly/our-insights/ upward-mobility-the-future-of-chinas-premium-car-market

3 Institute for Health Metrics and Evaluation's Global Burden of Disease Project and the Health Effects Institute (2017). State of Global Air 2017. Disponible en: https://www.stateofglobalair.org/sites/default/files/ SOGA2017_report.pdf
}

alrededor de 1,1 millones de muertes prematuras causadas por la contaminación del aire en 2015. Igualmente, un estudio de 2016 de la Escuela de Medio Ambiente de la Universidad de Nanjing señala que la contaminación está detrás de casi un tercio de las muertes en China. En este contexto, los Gobiernos locales están implementando restricciones a las nuevas matriculaciones. Al menos nueve ciudades en China han adoptado medidas para restringir el uso de vehículos de motor ${ }^{4}$ y seis de ellas han adoptado la decisión de restringir la compra de vehículos a sus ciudadanos. Todo ello con el objetivo de intentar limitar la cantidad de partículas contaminantes PM 2,5. Esto sitúa al desarrollo del vehículo eléctrico como uno de los principales retos del sector automovilístico en China y, al mismo tiempo, como una enorme oportunidad, pues este país es el principal dinamizador de esta industria a nivel mundial, ya que, además, domina el mercado de las baterías eléctricas. En este sentido, el Gobierno chino tiene previsto invertir 18.700 millones de USD entre 2011 y 2020 para convertirse en líder mundial en producción de vehículos eléctricos e híbridos, así como en sus principales componentes. No obstante, el éxito en el desarrollo del vehículo eléctrico pasará por la capacidad de desarrollo de una infraestructura de recarga a nivel internacional.

4. Reducción de los niveles de accidentalidad. Según datos de la Organización Mundial de la Salud, más de $200.000 \triangleright$

4 ChinaAutoWeb (2013, junio). More Chinese cities consider limiting car consumption. Disponible en: http://chinaautoweb.com/2013/06/ more-chinese-cities-consider-limiting-car-consumption/ 
personas pierden la vida cada año en las carreteras chinas, lo que posiciona al país como el segundo del mundo con mayor mortandad en carretera (solo superado por India). Además, destaca que la mayoría de los accidentes que se producen podrían evitarse. Aumentar los niveles de seguridad para reducir estas cifras se convierte así en uno de los principales retos que deberá abordar la industria automovilística del país.

5. Dispersión geográfica. En los próximos años, la dispersión geográfica será mucho mayor y sus características serán muy diferenciadas de unas regiones a otras. China pasará de demandar vehículos en las principales ciudades a demandarlos, en su mayoría, desde las ciudades de tercer y cuarto nivel. Un aspecto que, además, se ve favorecido por la mejora de la red de carreteras nacional.

6. Batalla por el talento. Cabe destacar la creciente demanda de profesionales formados de origen chino, especialmente a nivel del management, frente a la tendencia imperante de hace unos años, cuando la fuerza de trabajo en el sector era fundamentalmente extranjera. Es un reto actual para el Gobierno chino reducir la fuga de personal cualificado a otros países, generalmente europeos y americanos, mediante oportunidades laborales cualificadas locales.

\section{Oportunidades para las compañías españolas}

El mercado automovilístico chino representa una importante oportunidad para las compañías españolas del sector, en concreto para las empresas de componentes, las cuales, y a diferencia de los fabricantes de equipos originales (OEM), no están obligadas a asociarse con compañías locales para estar presentes en el mercado. Actualmente, los principales fabricantes de componentes tienen presencia en el país.

\section{El caso de Gestamp en China}

\subsection{Penetración en el mercado chino}

Gestamp es una multinacional española especializada en el diseño, desarrollo y fabricación de componentes metálicos de alta ingeniería para los principales fabricantes de automóviles. Desarrolla productos con un diseño innovador para conseguir vehículos cada vez más seguros y ligeros $y$, por tanto, mejores en relación al consumo de energía e impacto medioambiental. Sus productos abarcan las áreas de carrocería, chasis y mecanismos.

Desde su fundación en 1997, Gestamp basa su estrategia en dos pilares fundamentales. Por un lado, la incorporación de nuevas tecnologías en sus procesos de fabricación y el desarrollo de las tecnologías tradicionales. Por otro lado, la internacionalización, acompañando a sus clientes en sus procesos de expansión. Gestamp está presente actualmente en los principales núcleos de fabricación de automóviles a nivel mundial, con un total de 102 plantas industriales y 7 en construcción, 13 centros de I+D y una plantilla de más de 36.000 empleados repartidos en 21 países.

Dado el peso de China en la industria automovilística mundial, estar presente en el país era fundamental para cubrir las demandas de los fabricantes. Por ello, la estrategia de internacionalización de Gestamp, en los últimos $\triangleright$ 
años, ha situado a Asia como área prioritaria. Gestamp entró en el mercado chino en 2007, cuando inició la construcción de su primera planta productiva en la ciudad de Kushan, a $40 \mathrm{~km}$ de Shanghai. A finales de 2011, la compañía contaba en China con tres plantas de nueva construcción (greenfields) en Kushan, Wuhan y Chongqing, más dos provenientes del grupo alemán Edscha, adquirido en 2010; es decir, la de Shenyang se convertiría en su sexta planta productiva.

El tipo de negocio de Gestamp hace necesario contar con una proximidad con sus clientes, $y$ dado que alguno de ellos tenían plantas ubicadas en el nordeste de China y estas quedaban alejadas de los centros productivos que tenía Gestamp en el país, en noviembre de 2011 se decidió ubicar una nueva planta en su misma zona geográfica. En concreto, en la ciudad de Shenyang, que había experimentado un fuerte desarrollo en los últimos años gracias al apoyo del Gobierno central. Ubicación muy próxima a las plantas de BMW y de Volkswagen, lo que permitiría suministrarles componentes just in time. La inauguración oficial de la planta se produjo en 2013 y la primera producción en serie, en el mes de mayo.

Más recientemente (en noviembre de 2017), Gestamp ha inaugurado un nuevo centro de I+D en Shanghai, que ha supuesto una inversión de 2,5 millones de euros y que se suma al de la filial de mecanismos Edscha, que desarrolla sus actividades de I+D en Kunshan. El principal objetivo de esta nueva instalación es fortalecer la colaboración de Gestamp con los fabricantes de automóviles con presencia en China. Así, la Compañía busca codesarrollar junto a ellos para mejorar los procesos de fabricación, los productos y los costes.

Actualmente, Gestamp cuenta con nueve plantas y una en construcción en China, así como con dos centros de $1+D$, que en total suman aproximadamente 4.300 empleados en el país. En términos de ingresos, China es el cuarto mayor mercado de Gestamp, donde la compañía ha podido aumentar su facturación de un millón en 2008 a 719,6 millones de euros en 2016, principalmente como resultado de su crecimiento orgánico.

Cabe destacar que, para entrar en el mercado chino, Gestamp ha contado con el apoyo de la financiación de COFIDES. Adicionalmente, constatar que las fábricas de la compañía en China son de capital cien por cien propio, puesto que, como se ha mencionado anteriormente, la legislación del país no obliga a los fabricantes extranjeros de componentes para el sector a formar una alianza con socios locales, como sí que ocurre en el caso de los OEM (fabricantes de equipamiento original).

\subsection{Cercanía a los OEM}

La industria prevé que en los próximos años, hasta 2020, habrá una mayor tasa de crecimiento anual compuesto (CAGR) de ventas en Brasil, Rusia, India, China y en otras economías emergentes que la que habrá en economías maduras como las de Europa Occidental. Por ello, los OEM continúan desarrollando su presencia en estos mercados y, por tanto, aumentan su necesidad de establecer redes con proveedores de confianza situados en un área próxima. El incremento de la demanda del volumen de producción en estos mercados está en línea con una evolución de los estándares regulatorios e industriales que ya experimentaron anteriormente economías más maduras. Esta tendencia ofrece a los proveedores de primer nivel del sector de automoción, como Gestamp, una oportunidad de ampliar el negocio. 
Ante ello, la estrategia de Gestamp en China se centra en acompañar a sus clientes internacionales en su expansión, así como en aumentar la penetración de los OEM locales mediante el suministro de tecnología punta a todas las instalaciones que tiene en este país.

\subsection{La respuesta de Gestamp a los retos del mercado en China}

A continuación señalamos las principales respuestas de Gestamp a los retos del mercado chino:

1. Necesidad de cumplir con los estándares internacionales de calidad y seguridad. La compañía, tras años de experiencia, y gracias a la calidad que avala sus productos, se ha posicionado como proveedor de referencia en el diseño, desarrollo y fabricación de componentes. Por ello, Gestamp da respuesta a los estándares de la industria en esta materia.

2. La gama alta. Gestamp suministra componentes a los principales fabricantes mundiales de vehículos, entre los que se encuentran algunas de las principales marcas de alta gama del mercado como Daimler, Jaguar Land Rover o BMW.

3. Reducción de los niveles de accidentalidad. Gestamp basa su estrategia de futuro en la innovación, la l+D+i y el dominio tecnológico. La compañía desarrolla productos con un diseño innovador para conseguir vehículos cada vez más seguros y ligeros. En este sentido, la estampación en caliente junto a otras tecnologías del grupo permiten conseguir vehículos más seguros en piezas clave como las que conforman el habitáculo de seguridad del automóvil.

4. Vehículos impulsados por energías alternativas. Las tecnologías eléctricas e híbridas requieren vehículos más ligeros ante el peso de las baterías sobre el total de los automóviles. En este sentido, Gestamp ve como una oportunidad el hecho de aligerar, gracias a sus tecnologías, el peso de los vehículos eléctricos e híbridos. Además, Gestamp desarrolla y fabrica piezas como la caja de baterías, un elemento de vital importancia en este tipo de vehículos.

Adicionalmente, Gestamp eleva su compromiso con el medio ambiente en el proceso de fabricación (optimizando el uso de materia prima y mejorando la eficiencia de los procesos).

5. Batalla por el talento. Gestamp ha desarrollado un modelo propio para las implantaciones greenfield en el que se consideran, de manera integrada, tanto los aspectos relativos a la construcción de la planta como a la transferencia del conocimiento necesario. Cuando Gestamp entra en un nuevo mercado, apuesta por fomentar la contratación de empleados locales. Cuando no es posible encontrar perfiles profesionales en un determinado lugar, la compañía recurre a equipos de apoyo que durante un periodo de tiempo forman al personal local. En ocasiones, ante la escasez de profesionales en el entorno, Gestamp también colabora con agentes locales para capacitar a personas en habilidades técnicas y funcionales propias del sector de la automoción. Esta actividad no solo beneficia a Gestamp, sino que también contribuye a despertar el interés por la actividad $\triangleright$ 
industrial en la región fomentando la especialización de profesionales y siendo referente para otras empresas del sector en la zona.

\subsection{Contribución de la inversión de Gestamp en China}

En términos generales, la inversión de Gestamp en China contribuye de manera positiva al desarrollo industrial y social del país en diversos aspectos. Desde una perspectiva macroeconómica, cabe mencionar como principales impactos:

1. La contribución al PIB. Al tratarse de inversiones greenfield, se incrementa la capacidad productiva y el PIB de la economía china. Aún más importante, la inversión realizada va acompañada de la transferencia de tecnología avanzada en uno de los sectores considerados como prioritarios por el Gobierno chino, contribuyendo a la consolidación de los clusters industriales del país.

2. La creación de empleo. Gestamp, como hemos dicho anteriormente, cuenta actualmente con una plantilla de 4.300 empleados en China, cifra que se incrementará con la inauguración de la planta que actualmente tiene en construcción. A ello hay que añadirle la creación de empleo indirecto, derivada tanto de la construcción de la planta y sus instalaciones como de la propia actividad productiva de la planta.

3. Las diferentes Administraciones Públicas también se ven beneficiadas por los ingresos fiscales asociados al alquiler de los terrenos (de propiedad pública), a los impuestos, tasas y licencias pagados por la construcción de la nueva planta; y a los impuestos de la actividad empresarial ordinaria.

4. Impacto positivo en los proveedores locales productores de aceros tradicionales que mejoran sus procesos productivos para así conseguir estándares más altos de calidad.

5. Efecto demostración entre los competidores locales que permite la trasmisión de conocimiento y la mejora de sus procesos.

6. Contribución al desarrollo local, regional y nacional mediante la formación y capacitación mediante acuerdos de colaboración con diferentes centros de formación profesional, escuelas de negocios, universidades, etcétera. 